\title{
Emotion Ownership: Different Effects on Explicit Ratings and Implicit Responses
}

\author{
Peter Walla ${ }^{1 *}$, Lucy Rosser ${ }^{1}$, Janka Scharfenberger ${ }^{2}$, Cornelia Duregger ${ }^{1}$, \\ Shannon Bosshard ${ }^{1}$ \\ ${ }^{1}$ School of Psychology, Centre for Translational Neuroscience and Mental Health, University of Newcastle, \\ Newcastle, Australia \\ ${ }^{2}$ Faculty of Psychology, University of Vienna, Vienna, Austria \\ Email: "peter.walla@newcastle.edu.au
}

Received October $10^{\text {th }}, 2012$; revised November $16^{\text {th }}, 2012$; accepted December $15^{\text {th }}, 2012$

\begin{abstract}
This study is based on the idea that emotion-related processing happens on various different levels. Common methods that are used to measure different aspects of emotion-related processing exhibit specific sensitivities to one or the other of these separate processing levels. Here, the hypothesis was tested that explicit and implicit measures of emotion-related processing are differently influenced by self-referenced versus unreferenced emotion. The explicit measure was self reported valence and the implicit measure was startle reflex modulation. In one session, emotional scenes were paired with short sentences inducing self-reference (e.g. "this dog will attack you" written underneath the image of an aggressive dog), while in another session, emotional scenes were presented without any reference. During both sessions explicit as well as implicit responses were collected. Self-referenced unpleasant images were rated more negative and self-referenced pleasant images were rated more positive than images with no reference. In contrast, the implicit measure indicated greater startle responses related to self-reference regardless of emotion category. Under the common assumption that greater startle responses reflect increased affective negativity, this means that self-referenced pleasant images elicited more negative implicit affect than unreferenced pleasant images. However, in both cases (self-referenced and unreferenced) startle responses demonstrated valence depended modulation as expected. Thus, in our study startle responses demonstrated sensitivity to affective valence as well as self-reference. It is concluded that self-reference is linked to increased motivation, which in turn has been reported to be detectable via startle reflex modulation (SRM) as well.
\end{abstract}

Keywords: Emotion; Ownership; Self-Reference; Implicit Responses; Explicit Responses

\section{Introduction}

A new line of research follows the notion that there is more to emotion than subjective feeling. In their book chapter, Walla and Panksepp (2012) propose that an emotion is the consequence of affective processing and that affective processing can occur without generating an emotion. Based on a hierarchical mind model (e.g. Panksepp, 2011; Panksepp \& Solms, 2011; Walla \& Panksepp, 2012) there is at least three different processing levels on which affective processing can be influenced by simultaneous other processes. For example, one of these simultaneous processes can be self-reference. Self-reference is the process in which an individual, in this case a participant, is referred to within the context of a situation. Self-reference, or in other words mere ownership, (e.g. my garden) was found to elicit distinct brain activity, which is significantly different from brain activity reflecting somebody else's ownership (his garden; Walla et al., 2007, 2008). Only recently has the influence of self-reference on the processing of emotional words (e.g. my fear) been investigated with both electroencephalography (EEG) (Herbert et al., 2011a) and functional Magnetic Resonance Imaging (fMRI; Herbert et al., 2011b). Both studies revealed similar principle ownership effects as well as emotionspecific self-reference effects. In particular, words of a positive

${ }^{*}$ Corresponding author. nature elicited the strongest brain activities in the amygdala and the insular cortex when self referenced. Furthermore, a word recall task showed that self-referenced pleasant words were better recalled than unreferenced words. This indicates that affective content is differently processed depending on whether it is linked to the self or not.

The present study is meant to further investigate this issue by using emotionally evocative scenes. It is assumed that emotionally laden images more directly elicit emotion responses compared to emotion words. To elicit self-reference, images were shown simultaneously with short sentences. For example, participants were presented with an image of an aggressive dog together with text stating "This dog will attack you". For the current study, we hypothesised that the addition of self-reference would enhance self-reported valence in both directions. Thus, self-referenced pleasant scenes were expected to be rated as more pleasant compared to unreferenced pleasant scenes. Likewise, self-referenced unpleasant scenes were expected to be rated as more unpleasant compared to unreferenced unpleasant scenes. To test this hypothesis, valence ratings (and arousal ratings) were collected using the Self Assessment Manekin (SAM; Bradley \& Lang, 1994).

In addition to SAM, electromyography (EMG) was used to record eye blink-related muscle potential changes elicited through startle stimulation (startle reflex modulation; SRM). 
Previous literature has shown SRM to be sensitive to valence in numerous studies (Vrana et al., 1988; Lang et al., 1998; Walla, Brenner, \& Koller, 2011). Therefore, it is inferred that SRM is a suitable method to test whether possible changes in valence rating performance as a function of self-referenced versus unreferenced emotion images are associated with matching objective measures of valence. According to previous studies there is reason to believe that valence measures via startle reflex modulation do not necessarily match explicitly reported valence ratings (Geiser \& Walla, 2011; Junghöfer et al., 2010; Grahl et al., 2012). If such a discrepancy occurs in this study it may provide evidence to suggest that startle reflex modulation is also sensitive to aspects that are not necessarily valence-related. It has been reported that SRM is to some extent also sensitive to motivation (or arousal), but clear evidence does not exist. If it is the case that a discrepancy is found in our study, it may improve our understanding of what SRM truly measures. In addition, it would highlight that there is more to emotion than subjective feeling and finally that different measures are differently sensitive to the various aspects of emotion-related processing in the human brain.

\section{Method}

\section{Participants}

Data from 21 volunteers who participated in our study were collected. Mean age of all participants was 22.5 years $(\mathrm{SD}=4.7)$ (8 males). They were all University students, right-handed and had normal or corrected to normal vision. None of them had any neuropathological history. They all gave their informed consent and the project was approved by the ethics committee of the Newcastle University (Australia).

\section{Stimuli}

Emotion images were selected from the International Affective Picture System (IAPS; Lang, Bradley, \& Cuthbert, 2008). In particular, 90 images were used, of which thirty were unpleasant (mean pre-evaluated valence rating $=2.65(\mathrm{SD}=1.65)$, 30 were pleasant (mean pre-evaluated valence rating $=7.27$ $(\mathrm{SD}=1.69)$ and the remaining 30 were neutral (mean preevaluated valence rating $=4.51(\mathrm{SD}=1.92)$. Pleasant and unpleasant images had matching pre-evaluated arousal values, whereas neutral images were slightly less arousing. For each of the three emotion categories, 15 images were randomly assigned to the self-referenced group, whereas the remaining 15 images were kept unreferenced. Self-reference was induced by generating short sentences ( 3 to 5 words; e.g. "this surgery is on you" or "you won this money") that were displayed underneath the images. This set of 90 images was counterbalanced with respect to reference and presentation order to generate 6 different lists to be randomly used across all participants.

\section{Procedure}

On arrival at the laboratory participants were briefly screened regarding demographics and pathological history. They were seated on a comfortable chair and all sensors were attached while the experiment including all the tasks was explained. For startle reflex modulation, two electrodes were attached underneath the right eye (bipolar recording) and a ground electrode was placed on the left cheek. Explicit ratings (valence and arousal) were collected by using the SAM procedure (see. Bradley \& Lang, 1994). Participants viewed a screen (LCD monitor) on which all images (independent variables) were presented. Image presentation time was 5 seconds followed by a 1 second blank screen. Then, the self-assessment manekins for valence rating appeared (see Bradley \& Lang, 1994) until the response was given (using a computer mouse to click on one of the numbers between 1 and 9 which appeared on the screen) (maximum of 5 seconds). After the 1 second blank screen, the self-assessment manekins for arousal appeared until the response was given (again by clicking on one of the numbers between 1 and 9) (maximum of 5 seconds). The experiment was divided into two blocks with 45 images in each. In one of the blocks (counterbalanced) all images were associated with short sentences generating self-reference related to the emotion content of every image.

\section{Physiological Recordings and Data Processing}

Startle responses (eye blinks) were elicited by using a $50 \mathrm{~ms}$ burst of acoustic white noise $(105 \mathrm{~dB})$ delivered through headphones. Sound pressure level was controlled with a mobile measuring device (MicroAMP HA400 by Behringer). Via bipolar electromyography (EMG) carried out with the Nexus-10 mobile recording device from Mind Media BV muscle potential changes of the musculus orbicularis oculi of the left eye of every study participant were recorded. A dual channel electrode cable with carbon coating and active shielding technology for low noise and an additional ground electrode cable were used. EMG sampling rate was 2048 per s. A band pass filter from 20 $\mathrm{Hz}$ to $500 \mathrm{~Hz}$ was applied during online recording. Recorded data were stored on the hard drive of the laptop computer, which was used for data collection. Offline, raw EMG data were then recalculated by using the root mean square (RMS) method to transform EMG signals into amplitudes. All resulting amplitudes were visually inspected. In case of obviously bad signal-to-noise ratios respective data were not taken into account. Only a few missing values occurred, which were replaced by means of existing values from each respective condition. Finally, the resulting amplitudes (dependent variables) were log-transformed to partial out floor effects before they were subject to statistical analysis in terms of repeated measures ANOVA to calculate condition main effects and factor interactions (with SPSS version 19). Factors were affective category with two levels (pleasant and unpleasant) and factor reference with two levels (self reference and unreferenced).

\section{Results}

\section{Explicit Responses}

The mean self reported valence on a scale from 1 to 9 for the unpleasant affective category without self-reference was 2.43 $(\mathrm{SD}=.88)$. For the unpleasant affective category with selfreference the mean self reported valence was $1.87(\mathrm{SD}=.56)$. The mean self- reported valence for the pleasant affective category without self-reference was $5.85(\mathrm{SD}=.94)$ and for the pleasant affective category with self-reference it was 6.43 (SD $=1.06)$. The 2 ( 2 levels of affective category $) \times 2$ ( 2 levels of reference) repeated measures ANOVA revealed a significant affective category main effect $(p<.001)$. There is a non-significant reference main effect $(p=.897)$, which is not surprising, because self-reference had opposite effects on rating per- 
formance depending on affective category. However, a highly significant reference * affective category interaction occurs $(p$ $<.001$; Figure 1). Further analysis using t-tests revealed that for the unpleasant affective category, mean self-reported valence is significantly lower in the case of self-reference ( $p$ $<.001$ ), whereas for the pleasant affective category, mean self-reported valence is significantly higher in the case of selfreference $(p<.001)$. It can thus be inferred that self-reference increased affective intensity in both directions. Unpleasant affective content was rated more unpleasant and pleasant affective content more pleasant when the affective content was verbally referenced to the self of the observer.

\section{Implicit Responses}

The mean eye blink response for the unpleasant affective category without self-reference was $31.88 \mu \mathrm{V}(\mathrm{SD}=23.1)$ and with self-reference it was $33.5 \mu \mathrm{V}(\mathrm{SD}=21.7)$. For the pleasant affective category without self-reference the mean eye blink response was $29.7 \mu \mathrm{V}(\mathrm{SD}=22.5)$ and with self-reference it was $32.58 \mu \mathrm{V}(\mathrm{SD}=23.7)$ (Figure 2). A 2 (2 levels of affective category) $\times 2$ (2 levels of reference) repeated measures

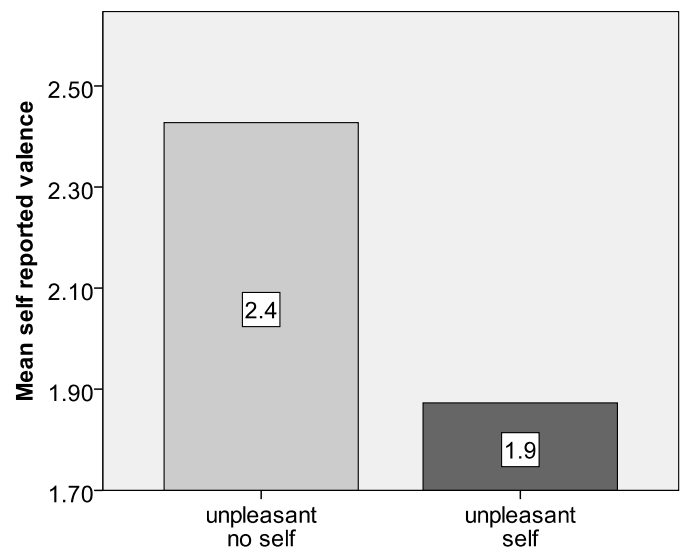

(a)

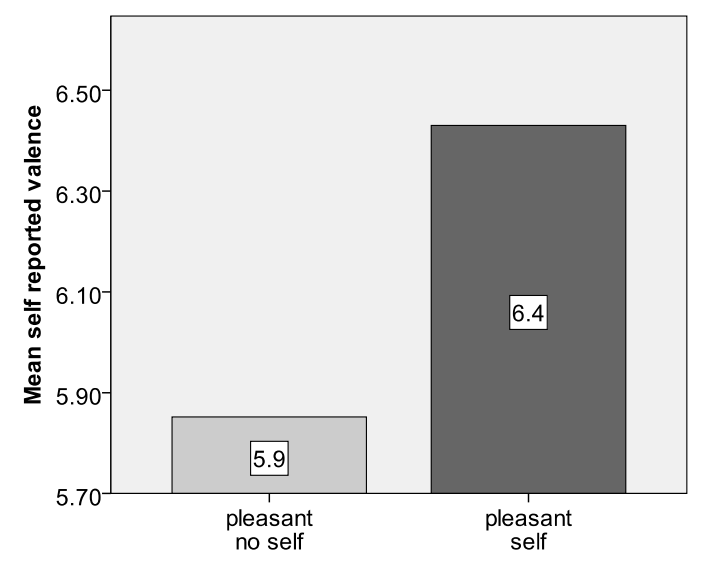

(b)

Figure 1.

(a) Depicts the valence associated with unpleasant images when a self-referenced sentence is present or not (the greater the mean selfreported valence, the more positive the emotion). Similarly, (b) illustrates the explicit ratings of pleasant images with and without the inclusion of a self-referenced sentence.

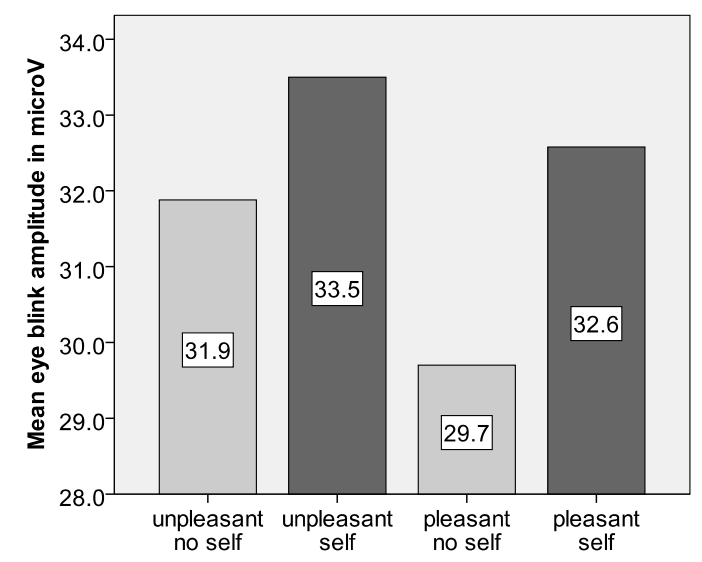

Figure 2.

This bar graph demonstrates the sensitivity of SRM to not only differentiate between the emotional response associated with pleasant and unpleasant images, but also its ability to detect the effect of self-reference regardless of valence.

ANOVA of log-transformed values revealed a significant affective category main effect $(p=.049)$. A significant reference main effect $(p=.019)$ was also found, but no significant reference $*$ affective category interaction occurred $(p=.473)$. These results demonstrate that self-reference as well as valence category modified eye blink responses. Follow-up t-tests revealed that self-reference modified eye blink responses significantly only for the pleasant affective category $(p=.030)$, whereas for the unpleasant affective category this effect was not significant $(p=.233)$.

\section{Discussion}

The current study revealed that participants were seen to rate pleasant images as more pleasant and unpleasant images as more unpleasant when these were self-referenced. For both valence categories these effects were statistically significant. However, although pleasant images were rated more pleasant when self-referenced, eye blink responses were potentiated indicating greater affective negativity. Given that eye blink responses are sensitive in detecting deep affective processes (Vrana et al., 1988) it was unexpected to find that the explicit/behavioural output showed contradictory effects. Only for the unpleasant valence category did we find a match between explicit rating and eye blink responses, because both measures revealed increased affective negativity for self-referenced images.

Discrepancies between explicit ratings and objective measures related to affective content have been described earlier. For example, Mühlberger et al. (2008) found that explicit pleasantness ratings, while virtually driving through a dark tunnel, did not always match simultaneously registered startle responses. Dunning et al. (2010) found a non-linear relationship between the intensity of angry faces and startle eye blink responses. Similarly, Walla et al. (2010) found that explicitly stated food preference did not always match startle responses. Geiser and Walla (2011) showed that virtually walking through urban environments can result in different effects depending on explicit or implicit measures. A recent study revealed that even specific bottle-shapes can alter startle responses without changing explicit rating performance (Grahl et al., 2012). It is suggested that such discrepancies are due to cognitive processing associ- 
ated with explicit responses that interfere with raw affective processing. In other words, the valence of cognitively appraised affective content can be different to the valence of the actual underlying raw affective content itself. This idea fits well the emotion-model as proposed by Walla and Panksepp (2012).

Alternatively, one might think that in the current study, eye blink responses were more reflective of motivation-related aspects of affective content than valence-related aspects. After all, the effect of mere self-reference on startle responses did not depend on condition of emotion. In the pleasant as well as the unpleasant emotion condition, self-reference resulted in enhanced eye blink amplitudes. It may be assumed that motivational aspects are more pronounced in cases of self-reference regardless of valence. In fact, it has been reported that SRM provided an insight into not only the emotional responses toward images, but also motivation related aspects related to images (Lang et al., 1998; Gard et al., 2007; Grillon \& Baas, 2003). In contrast, some authors tended to focus more heavily on the emotional aspects that SRM is able to measure. This study revisits the findings of previous literature and supports the use of SRM as not only a measure of emotion but also a measure of motivation. This seems reasonable assuming that self-reference is directly correlated with motivation. The more an individual's self is linked with pleasant or unpleasant input the higher the motivation to become active.

Finally though, it must be emphasised that SRM still presented the expected valence-related difference between pleasant and unpleasant images. Regardless of whether self-referenced or not, pleasant images revealed reduced eye blink responses and negative images resulted in larger eye blink amplitudes. In sum, this study provides evidence that SRM is indeed sensitive to both affective valence and self-reference. Finally, we conclude that the present study supports the idea that the various methods that are used to investigate emotion-related processing are differently sensitive to different aspects of emotion-related processing. Due to its objective nature (independent from self report) it is believed that SRM will be used in various further applied fields in the future (see Koller \& Walla, 2012; ArthurKelly et al., 2012).

\section{REFERENCES}

Arthur-Kelly, M., Lyons, G. S., \& Walla, P. (2012). Toward improved ways of knowing children with multiple disabilities (PMD): Introducing startle reflex modulation. Developmental Neurorehabilitation, in press.

Bradley, M. M., \& Lang, P. J. (1994). Measuring emotion: The selfassessment manikin and the semantic differential. Journal of Behavioural Therapy and Experimental Psychiatry, 25, 49-59. doi:10.1016/0005-7916(94)90063-9

Cuthbert, B. N., Schupp, H. T., Bradley, M., McManis, M., \& Lang, P. J. (1998). Probing affective pictures: Attended startle and tone probes. Psychophysiology, 35, 344-347. doi:10.1017/S0048577298970536

Dunning, J. P., Auriemmo, A., Castille, C., \& Hajcak, G. (2010). In the face of anger: Startle modulation to graded facial expressions. Psychophysiology, 47, 874-878.

Gard, D. E., Gard, M. G., Mehta, N., Kring, A. M., \& Patrick. C. J. (2007). Impact of motivational salience on affect modulated startle at early and late probe times. International Journal of Psychophysiology, 66, 266-270. doi:10.1016/j.ijpsycho.2007.05.005
Geiser, M., \& Walla, P. (2011). Objective measures of emotion during virtual walks through urban environments. Applied Sciences, 1, 1-11.

Grahl, A., Greiner, U., \& Walla, P. (2012). Bottle shape elicits genderspecific emotion: A startle reflex modulation study. Psychology, 3, 548-554. doi:10.4236/psych.2012.37081

Grillon, C., \& Baas, J. (2003) A review of the modulation of the startle reflex by affective states and its application in psychiatry. Clinical Neurophysiology, 114, 1557-1579. doi:10.1016/S1388-2457(03)00202-5

Herbert, C., Herbert, B. M., Ethofer, T., \& Pauli, P. (2011a) His or mine? The time course of self-other discrimination in emotion processing. Social Neuroscience, 6, 277-288. doi:10.1080/17470919.2010.523543

Herbert, C., Herbert, B. M., \& Pauli, P. (2011b). Emotional self-reference: Brain structures involved in the processing of words describing one's own emotions. Neurophsycologia, 49, 2947-2956. doi:10.1016/j.neuropsychologia.2011.06.026

Jackson, D. C., Malmstadt, J. R., Larson, C. L., \& Davidson, R. J. (2000). Suppression and enhancement of emotional responses to unpleasant pictures. Psychophysiology, 37, 515-522. doi:10.1111/1469-8986.3740515

Junghöfer, M., Kissler, J., Schupp, H. T., Putsche, C., Elling, L., \& Dobel, C. (2010). A fast neural signature of motivated attention to consumer goods separates the sexes. Frontiers in Human Neuroscience, 4, 179. doi:10.3389/fnhum.2010.00179

Koller, M., \& Walla, P. (2012). Measuring affective information processing in information systems and consumer research - Introducing startle reflex modulation. ICIS 2012 Proceedings, in press.

Lang, P. J., Bradley, M. M., \& Cuthbert, B. N. (2008). International affective picture system (IAPS): Affective ratings of pictures and instruction manual. Technical Report A-8, Gainesville, FL: University of Florida,.

Lang, P. J., Bradley, M. M., \& Cuthbert, B. N. (1998). Emotion, motivation, and anxiety: Brain mechanisms and psychophysiology. Biological Psychiatry, 44, 1248-1263. doi:10.1016/S0006-3223(98)00275-3

Mühlberger, A., Wieser, M. J., \& Pauli, P. (2008). Darkness-enhanced startle responses in ecologically valid environments: A virtual tunnel driving experiment. Biological Psychology, 77, 47-52. doi:10.1016/j.biopsycho.2007.09.004

Vrana S R, Spence E L \& Lang P J (1988) The startle probe response: a new measure of emotion? Journal of Abnormal Psychology, 97, 487491. doi:10.1037/0021-843X.97.4.487.

Walla, P., \& Panksepp, J. (2013). Neuroimaging helps to clarify affective processing without necessarily clarifying emotions. In $\mathrm{K}$. N. Fountas, (Ed.), Novel frontiers of advanced neuroimaging. Intech. http://www.intechopen.com/books/novel-frontiers-of-advanced-neur oiming/neuroimaging-helps-to-clarify-brain-affective-processingwith out-necessarily-clarifying-emotions

Walla, P., Brenner, G., \& Koller, M. (2011). Objective measures of emotion related to brand attitude: A new way to quantify emotionrelated aspects relevant to marketing. Plos One, 6, e26782. doi:10.1371/journal.pone.0026782

Walla, P., Richter, M., Färber, S., Leodolter, U., \& Bauer, H. (2010). Food evoked changes in humans: Startle response modulation and event-related potentials (ERPs). Journal of Psychophysiology, 24, 25-32. doi:10.1027/0269-8803/a000003

Walla, P., Duregger, C., Greiner, K., Thurner, S., \& Ehrenberger, K. (2008). Multiple aspects related to self awareness and the awareness of others: An electroencephalography (EEG) study. Journal of Neural Transmission, 115, 983-992. doi:10.1007/s00702-008-0035-6

Walla, P., Greiner, K., Duregger, C., Deecke, L., \& Thurner, S. (2007). Self-awareness and the subconscious effect of personal pronouns on word encoding: A magnetoencephalography (MEG) study. Neuropsychologia, 45, 796-809. doi:10.1016/j.neuropsychologia.2006.08.017 